\title{
HeI emission line formation in symbiotic stars and novae
}

\author{
H.M. Schmid, \\ Institute of Astronomy, ETH Zentrum, CH-8092 Zürich, Switzerland
}

\section{Observational data}

The HeI triplet to singlet line ratio $\Lambda=F(\lambda 5876) / F(\lambda 6678)$ in symbiotic stars and novae is often much lower than the planetary nebulae value of $\approx 3.5$, which is also the low density value $\Lambda_{0}$ predicted by recombination theory.

Fig.1 shows that the dereddened HeI line ratios $\Lambda$ are different for $\mathrm{S}$ type and D type symbiotic stars. The spectrophotometric data are mainly from Allen (1984, personal communication) and Blair et al.(1983). The S and D type classification divides the symbiotic stars according to the IR continuum into objects with a stellar continuum or dust emission. Practically all D types have low nebular densities $N_{e} \lesssim 10^{7} \mathrm{~cm}^{-3}$ and a rich spectrum of forbidden lines, while in $\mathrm{S}$ type objects these lines are suppressed by collisional deexcitation due to the high electron densities $N_{e} \gtrsim 10^{7} \mathrm{~cm}^{-3}$. The observed HeI line ratio $\Lambda$ in $\mathrm{S}$ type symbiotics, which is systematically too low when compared with the theoretical low density value $\Lambda_{0}=3.5$, can therefore be attributed to the high nebular densities in these objects.

Low HeI line ratios $\Lambda \approx 2$ are also observed in novae. In Fig. 2 the line ratio $\Lambda$ is plotted as a function of time for the fast Nova Cyg 1975 (data from Ferland et al.,1986). $\Lambda$ is lowest for the highest nebular densities shortly after outburst.

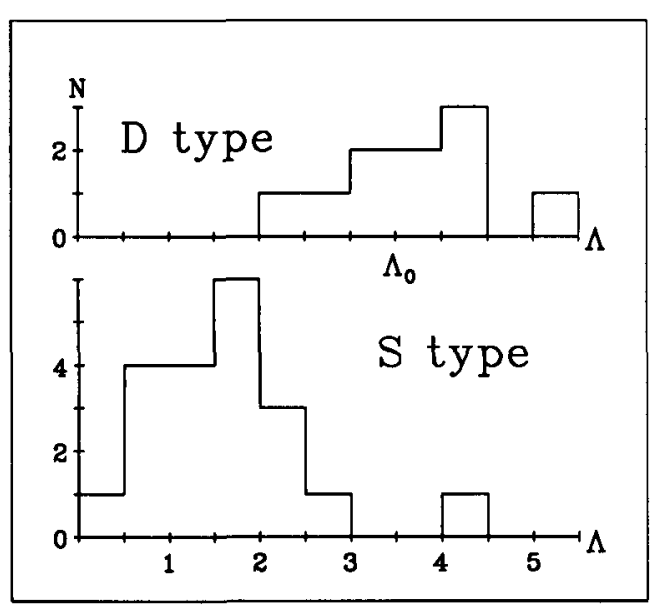

Fig.1. Distribution of the HeI line ratio $\Lambda$ in $D$ type and $S$ type symbiotic stars.

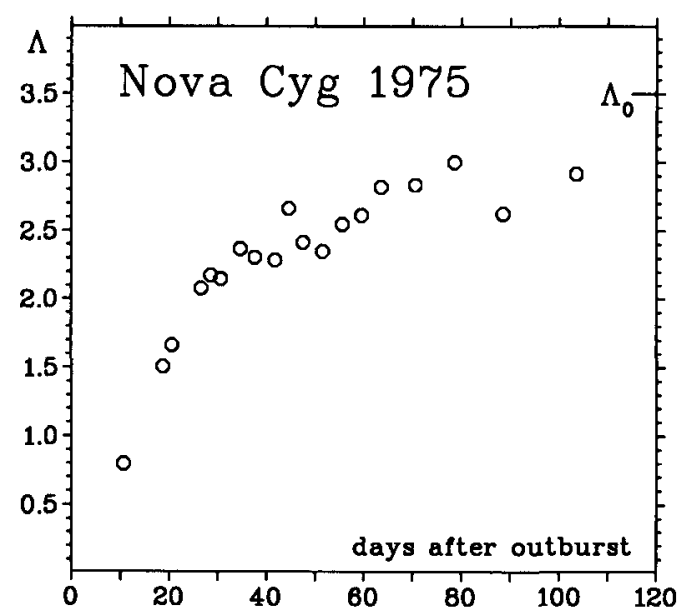

Fig.2. Evolution of the HeI line ratio $\Lambda$ in Nova Cyg 1975. 
As the envelope expands and $N_{e}$ decreases, the line ratio evolves towards the low density value $\Lambda_{0}$.

These data suggest that the HeI line ratio $\Lambda=F(\lambda 5876) / F(\lambda 6678)$ in symbiotic stars and novae depends strongly on nebular density.

\section{Calculations}

The line formation in $\mathrm{HeI}$ is complicated by the metastability of the levels $2{ }^{3} \mathrm{~S}$ and $2{ }^{1} \mathrm{~S}$. To investigate in detail the Hel line formation in dense nebulae the coupled rate equations for a 17 level $\mathrm{HeI}$ atom have been solved. Fig. 3 shows calculated line ratio $\Lambda=F(\lambda 5876) / F(\lambda 6678)$ as a function of $N_{e}$ for case B (optical depths $\tau=\infty$ in the HeI Lyman lines $1^{1} \mathrm{~S}-n^{1} \mathrm{P}$ ) and neglecting line transfer effects among the excited levels. According to the line ratios $\Lambda=\Lambda_{0}, \Lambda>\Lambda_{0}$ and $\Lambda<\Lambda_{0}$ three density regimes can be distinguished:

(a) Nebulae with low densities $N_{e} \lesssim 10^{3} \mathrm{~cm}^{-3}$ where no collisions need be considered.

(b) Nebulae with intermediate densities $10^{3} \mathrm{~cm}^{-3} \lesssim N_{e} \lesssim 10^{7} \mathrm{~cm}^{-3}$, like planetary nebulae, where collisional enhancement of $\mathrm{HeI}$ emission lines from $2^{3} \mathrm{~S}$ is important (e.g. Clegg,1987). As collision strengths for spin changing transitions are much smaller than transitions among the same spin, the triplet to singlet line ratio $\Lambda=F(\lambda 5876) / F(\lambda 6678)$ is enhanced if collisions from $2^{3} \mathrm{~S}$ take place.

(c) Nebulae which have high densities $N_{e} \gtrsim 10^{7} \mathrm{~cm}^{-3}$ as in $\mathrm{S}$ type symbiotic stars and novae. In these objects also collisions from $2^{1} \mathrm{~S}$ must be included. Because the radiative decay for $2^{1} \mathrm{~S}$ is more than 5 orders of magnitude faster than for $2^{3} \mathrm{~S}$, this effect can be neglected in low and intermediate density nebulae. Collisions from $2^{1} \mathrm{~S}$ enhance mainly the singlet lines and cause the low HeI line ratio $\Lambda=$ $F(\lambda 5876) / F(\lambda 6678)$ as seen in the observational data presented in the previous section.

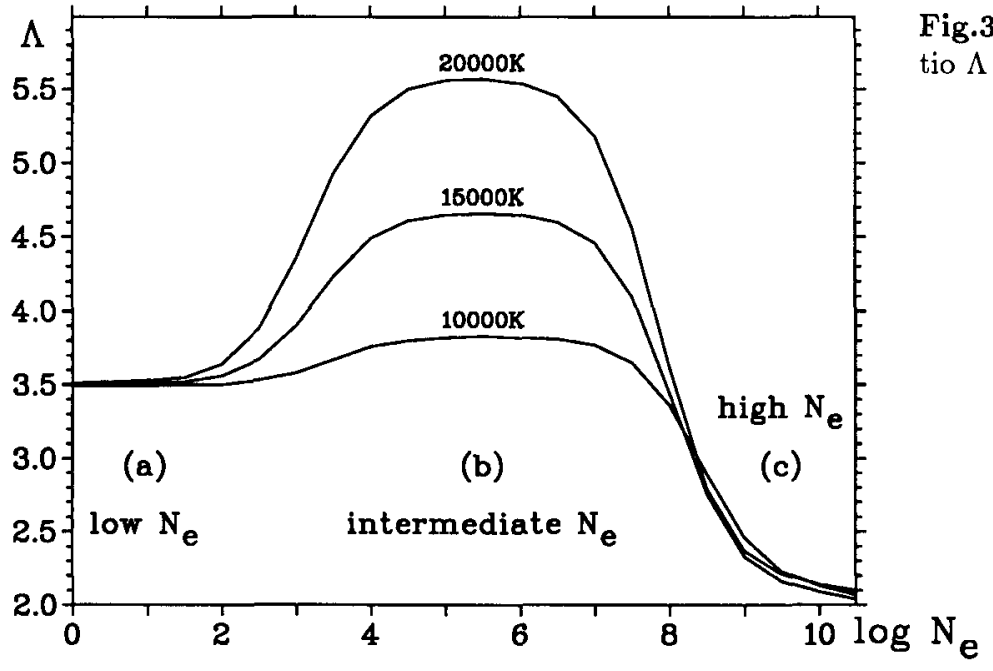


If case $\mathrm{A}(\tau=0)$ is assumed for HeI Lyman lines, the observed line ratios $\Lambda$ can not be reproduced. The population of the metastable level $2^{1} \mathrm{~S}$ is too low for collisional enhancements, because depopulation via collisional excitations (mainly to $2^{1} \mathrm{P}$ ) and cascades to the ground state are very effective. Observations of symbiotic stars and novae therefore suggest that conditions for the HeI Lyman lines must be close to case B.

\section{Conclusion}

The HeI line ratio $\Lambda=F(\lambda 5876) / F(\lambda 6678)$ can be used for nebular diagnostics. Fig. 3 shows that in the intermediate density range $\Lambda$ is a function of the nebular temperature $T_{e}$. Additionally, nebulae with low or intermediate densities $N_{e} \lesssim 10^{7} \mathrm{~cm}^{-3}$ or high densities $N_{e} \gtrsim 10^{7} \mathrm{~cm}^{-3}$ can be distinguished according to the observed HeI line ratio $\Lambda \gtrsim \Lambda_{0}$ or $\Lambda<\Lambda_{0}$ respectively.

For $\mathrm{He}^{+}$abundance determination the calculated HeI recombination line emissivities must include collisional contributions. Clegg (1987) gives for several HeI lines the ratio of excitations by collisions to recombinations $C / R$ for intermediate density nebulae. But in high density nebulae $N_{e} \gtrsim 10^{7} \mathrm{~cm}^{-3}$ and under case B conditions, the radiative decay rate $2{ }^{1} \mathrm{~S}-1{ }^{1} \mathrm{~S}$ becomes slow with respect to collisional rates and the population of $2^{3} \mathrm{~S}$ and $2^{1} \mathrm{~S}$ rises further. As a result the $C / R$ ratios are much larger in high density nebulae. This can explain very strong $\mathrm{HeI}$ lines seen in some symbiotic stars without postulating helium overabundances.

Acknowledgements. I thank D. Allen for providing spectrophotometric data and $H$. Nussbaumer and M. Vogel for discussions and help.

References.

Allen, D.A.: 1984, Proc. Astr. Soc. Aust. 5, 369

Blair, W.P, Stencel, R.E., Feibelman, W.A., Michalitsianos, A.G.: 1983, Astrophys. J. Suppl. Ser. 53, 573

Clegg, R.E.S.: 1987, Mon. Not. R. astr. Soc. 229, 31P

Ferland, G.J., Lambert, D.L., Woodman, J.H.: 1986, Astrophys. J. Suppl. Ser. 60,375 